\title{
TRAUMA ABDOMINAL FECHADO: ANÁLISE DOS PACIENTES VÍTIMAS DE TRAUMA HEPÁTICO EM UM HOSPITAL UNIVERSITÁRIO DE CURITIBA
}

\section{BLUNT ABDOMINAL TRAUMA: ANALYSIS OF VICTIMS OF HEPATIC TRAUMA IN AN UNIVERSITY HOSPITAL OF CURITIBA}

\author{
Luiz Carlos von Bahten, TCBC-PR ${ }^{1}$; João Eduardo Nicoluzzi, TCBC-PR²; Márcia Olandoski³; \\ Carlos Andrés Rodriguez Pantanali ${ }^{4}$; Ruy Fernando Kuenzer Caetano da Silva
}

\begin{abstract}
RESUMO: Objetivo: Determinar as características comuns referentes ao mecanismo de trauma, lesões anatômicas e fisiológicas, dor abdominal e à fratura de costelas das vítimas de trauma hepático contuso, no momento da admissão hospitalar. Método: Revisão dos prontuários de dezembro de 1999 a janeiro de 2003, e selecionados 524 através do Software TNVT Plus (versão 2.0.0.213 - ano 1996), admitidos no Serviço de Emergência, vítimas de trauma tóraco-abdominal contuso e posteriormente internados, que apresentavam hepatectomia parcial, hepatorrafia, laparotomia exploradora, redução de fratura de costelas ou tratamento conservador de fratura de costelas. Foram excluídos aqueles que apresentavam trauma penetrante, que foram a óbito na sala de emergência e os menores de 16 anos, restando 200 prontuários. Resultados: Considerando todos os traumas hepáticos, 37,63\% foram causados por acidentes com veículo automotor, sendo que os traumas por esse mecanismo provocaram 54,29\% das lesões mais graves (grau III, IV e V). Estas lesões causaram dor abdominal em 75,86\% dos casos, e as mais leves (grau I e II), em 69,70\%. A freqüência cardíaca e a pressão arterial sistólica média dos pacientes com lesões grau I ou II, foram, respectivamente, 100,37 batimentos por minuto e 114,45 mmHg, e naqueles com lesões grau III, IV, ou V, foram 105,69 batimentos por minuto e 107,88 $\mathrm{mmHg}$, respectivamente. A fratura de costela à direita associou-se à lesão hepática grau I ou II em 29,41\% dos casos. Conclusão: Acidentes por veículo automotor foram a causa mais comum de trauma hepático e o mecanismo de trauma que provocou as lesões mais graves. Estas lesões causaram dor abdominal com a mesma freqüência que as mais leves. A freqüência cardíaca e a pressão arterial sistólica média não possuíram relação com o grau da lesão hepática e a fratura de costelas à direita influenciou apenas na presença da lesão hepática. (Rev. Col. Bras. Cir. 2005; 32(6): 316-320).
\end{abstract}

Descritores: Traumatismos abdominais; Fígado/ lesões; Ferimentos e lesões.

\section{INTRODUÇÃO}

O fígado é um dos órgãos intra-abdominais mais acometidos no trauma pelo seu tamanho e sua localização anatômica ${ }^{1,2}$, ocorrendo lesão hepática em $20 \%$ dos pacientes vítimas de trauma contuso ${ }^{2}$. Nestes casos a lesão hepática é decorrente de impacto direto, compressão entre o rebordo costal direito e a coluna vertebral ou devido às forças de desaceleração. A lesão isolada do fígado ocorre em somente $10 \%$ dos pacientes, havendo, na maioria dos casos de trauma hepático, lesões de outros órgãos e vísceras ${ }^{3}$.

Dos lobos hepáticos, o direito é o mais comumente afetado em virtude de ser a porção do parênquima hepático mais volumoso ${ }^{3,4}$. As lesões do lobo esquerdo, por sua vez, são raras e, quando presentes, geralmente estão associadas com impacto direto no abdome superior, como no atropelamento, em que a roda causa compressão no tórax e no abdome. Já as lesões do lobo caudado são extremamente raras e geralmente estão acompanhadas por traumas de outros órgãos ${ }^{4}$.

Nos últimos 15 anos, houve uma dramática mudança na conduta frente ao trauma hepático contuso, especialmente com o auxílio de exames de imagem e o surgimento do tratamento não operatório. Atualmente, o tratamento conservador é utilizado em mais de $80 \%$ dos traumas hepáticos contusos, sendo considerado um método seguro e eficaz 5 . A falha neste tipo de conduta geralmente é causada por lesões abdominais associadas 6 .

O tratamento conservador do trauma hepático fechado apresenta vantagens em relação ao operatório, como a menor necessidade de transfusão sanguínea, menor ocorrência de sepse intra-abdominal, menor necessidade de cuidados intensivos ${ }^{7}$ e menor mortalidade ${ }^{8}$.

O presente estudo teve por objetivo determinar as características comuns referentes ao mecanismo de trauma, às lesões anatômicas e fisiológicas, à dor abdominal e à fratura de costelas dos pacientes vítimas de trauma hepático contuso, no momento da admissão hospitalar.

\section{MÉTODO}

Este estudo foi realizado no SAME (Serviço de Arquivo Médico e Estatístico) do Hospital Universitário Cajuru,

\footnotetext{
1. Professor Titular e Coordenador da Disciplina de Cirurgia do Trauma da PUC-PR; Professor Adjunto da Disciplina de Cirurgia do Trauma da UFPR; Doutor em Clínica Cirúrgica pela UNICAMP-SP

2. Professor Adjunto da Disciplina de Cirurgia do Trauma pela PUCPR; Doutor em Clínica Cirúrgica pela UFPR.

3. Professora Adjunta da Disciplina de Bioestatística da PUC-PR.

4. Acadêmicos de Medicina da PUC-PR.
}

Recebido em 11/08/2005

Aceito para publicação em 11/10/2005

Conflito de interesse : nenhum

Fonte de financiamento: nenhuma

Trabalho realizado na Disciplina de Cirurgia do Trauma da Pontifícia Universidade Católica do Paraná. 
Tabela 1 - Prevalência dos mecanismos de trauma.

\begin{tabular}{lcc}
\hline Mecanismo do trauma & Freqüência & Percentual \\
\hline Agressão & 19 & 9,50 \\
Atropelamento & 67 & 33,50 \\
Queda de nível & 21 & 10,50 \\
Veículo auto-motor & 93 & 46,50 \\
Total & 200 & 100,00 \\
\hline
\end{tabular}

Tabela 2 - Prevalência dos graus de lesão hepática.

\begin{tabular}{ccc}
\hline Grau da lesão hepática & Freqüência & Percentual \\
\hline I & 10 & 15,15 \\
II & 24 & 36,36 \\
III & 15 & 22,72 \\
IV & 14 & 21,22 \\
V & 3 & 4,55 \\
VI & 0 & 0,00 \\
Total & 66 & 100,00 \\
\hline
\end{tabular}

no período de janeiro a maio de 2003, segundo as Diretrizes e Normas Regulamentadoras de Pesquisas Envolvendo Seres Humanos do Conselho Nacional de Saúde (Resolução 196/96).

Foi realizada a revisão dos prontuários de dezembro de 1999 a janeiro do ano 2003, e selecionados, através do Software TNVT Plus (versão 2.0.0.213 - ano 1996) aqueles que apresentaram pelo menos um dos seguintes procedimentos:

- Hepatectomia parcial;

- Hepatorrafia;

- Laparotomia exploradora;

- Redução de fratura de costelas;

- Tratamento conservador de fratura de costelas.

Foram selecionados 524 prontuários, dos quais foram retirados aqueles que não apresentavam mecanismo de trauma contuso, que foram a óbito na sala de emergência e os pacientes menores de 16 anos. Os dados desses prontuários foram coletados de forma retrospectiva e as variáveis de interesse no estudo foram: etiologia do trauma, órgão lesado, grau e segmento da lesão, freqüência cardíaca e pressão arterial sistólica média na admissão do paciente ao hospital e fraturas de costela.

As variáveis quantitativas foram expressas por médias e desvios padrões e as variáveis categóricas por frequiências e percentuais. Para comparar os mecanismos de trauma em relação às variáveis quantitativas, foi utilizada a análise de variância e para avaliar os mecanismos de trauma em relação a variáveis categóricas, foi usado o teste de Qui-quadrado.

Na comparação dos graus de lesão em relação a variáveis quantitativas, foi utilizado o teste t de Student para amostras independentes e na comparação dos graus de lesão em relação a variáveis categóricas, o teste de Qui-quadrado ou o teste exato de Fisher. Em todos os testes, um valor de $\mathrm{p}<0,05$ foi considerado estatisticamente significante.

Para as variáveis pressão arterial (PA) sistólica e freqüência cardíaca (FC), no momento da admissão do paciente ao hospital (entrada), testou-se a hipótese nula de que as médias são iguais para os graus de lesão I, II ou III e para os graus de lesão IV, V ou VI, versus a hipótese alternativa de médias diferentes. Para tanto, foi utilizado o testes t de Student.

\section{RESULTADOS}

Dos mecanismos de trauma, houve maior incidência de traumas por veículo auto-motor (46,5\%), seguida pelos atropelamentos $(33,5 \%)$, e menor por agressão $(9,5 \%)$ (Tabela 1$)$.

Em relação ao grau de lesão hepática, houve maior incidência de lesão grau II (36,36\%), seguida de lesão grau III $(22,72 \%)$ e menor incidência de lesão graus V $(4,55 \%)$ e VI (0\%) (Tabela 2).

Observou-se que a lesão hepática grau II foi a mais prevalente nos traumas causados por veículo auto-motor $(37,14 \%)$ e por atropelamento $(31,82 \%)$. Verificou-se também que os acidentes por veículo auto-motor provocaram a maioria das lesões hepáticas, tanto as mais leves (até grau II) quanto as mais graves (graus III, IV e V). (Tabela 3).

Quanto aos mecanismos de trauma, houve maior incidência de trauma hepático em virtude de acidentes por veí-

Tabela 3 - Relação entre os graus de lesão hepática e o mecanismo de trauma.

\begin{tabular}{ccccc}
\hline $\begin{array}{c}\text { Grau de } \\
\text { lesão hepática }\end{array}$ & Agressão & Atropelamento & Queda de nível & Veículo auto-motor \\
\hline I & 2 & 4 & 1 & 3 \\
& $50,00 \%$ & $18,18 \%$ & $20,00 \%$ & $8,57 \%$ \\
II & 2 & 7 & 2 & 13 \\
& $50,00 \%$ & $31,82 \%$ & $40,00 \%$ & $37,14 \%$ \\
III & 0 & 6 & 0 & 9 \\
& $0,00 \%$ & $27,27 \%$ & $0,00 \%$ & $25,72 \%$ \\
IV & 0 & 5 & 2 & 7 \\
& $0,00 \%$ & $22,73 \%$ & $40,00 \%$ & $20,00 \%$ \\
V & 0 & 0 & 0 & 3 \\
& $0,00 \%$ & $0,00 \%$ & $0,00 \%$ & $8,57 \%$ \\
Total & 4 & 22 & 5 & 35 \\
\hline
\end{tabular}


Tabela 4 - Relação entre a presença de trauma hepático e os mecanismos de trauma.

\begin{tabular}{lcccl}
\hline Fígado & Agressão & Atropelamento & Queda de nível & Veículo auto-motor \\
\hline Não & 15 & 45 & 16 & 58 \\
& $78,95 \%$ & $67,16 \%$ & $76,19 \%$ & $62,37 \%$ \\
Sim & 4 & 22 & 5 & 35 \\
& $21,05 \%$ & $32,84 \%$ & $23,81 \%$ & $37,63 \%$ \\
Total & 19 & 67 & 21 & 93 \\
\hline
\end{tabular}

Teste Qui-Quadrado: $p=0,4021$.

Tabela 5 - Relação entre a dor abdominal e o grau de lesão hepática.

\begin{tabular}{llc}
\hline Dor abdominal & \multicolumn{2}{c}{ Grau de lesão hepática } \\
\cline { 2 - 3 } & Grau I ou II & Grau III, IV ou V* \\
\hline Não & 10 & 7 \\
& $29,41 \%$ & $21,88 \%$ \\
Sim & 24 & 25 \\
& $70,59 \%$ & $78,12 \%$ \\
Total & 34 & 32 \\
\hline
\end{tabular}

Teste Exato de Fisher: $p=0,5783$

*Não ocorreram lesões hepáticas grau VI.

culo auto-motor $(37,63 \%)$ e menor incidência por agressão $(21,05 \%)$. (Tabela 4).

Correlacionando-se a dor abdominal e o grau de lesões hepáticas, verificou-se que, tanto a maioria das lesões leves $(70,59 \%)$ quanto a maioria das mais graves $(78,12 \%)$, apresentavam este sintoma (Tabela 5).

Analisando-se o grau de lesões hepáticas e a fratura de arcos costais à direita, observou-se que presença dessas fraturas estava associada às lesões hepáticas grau I ou II em $29,41 \%$ dos casos.

No momento da admissão do paciente ao hospital (entrada), a frequiência cardíaca média e a pressão arterial sistólica não variaram significativamente de acordo com os graus de lesão hepática (Tabela 6).

\section{DISCUSSÃO}

No trauma de fígado, as lesões mais leves (até grau III) correspondem à maioria $(85 \%)$ de todas as lesões hepáti$\operatorname{cas}^{1,3}$. No presente estudo, também se verificou maior incidên- cia destes graus de lesões $(74,23 \%)$. Sempre que se estuda trauma abdominal, o mecanismo de trauma é um dos fatores determinantes para levantar a suspeita dos prováveis órgãos acometidos, bem como do grau da lesão desses órgãos. Croce et al..$^{9}$ em estudo com 136 pacientes, observaram que $95 \%$ das lesões hepáticas foram decorrentes de acidentes por veículos auto-motores. Pachter et al. ${ }^{10}$ por sua vez, em estudo multicêntrico com 404 pacientes, encontraram $72 \%$ das lesões de fígado secundárias a este mesmo mecanismo de trauma. No presente estudo, em concordância com a literatura, verificouse que $70,47 \%$ (atropelamento mais veículo auto-motor) das lesões hepáticas foram decorrentes desse mecanismo de trauma (Tabela 4).

Observou-se também que as lesões hepáticas graves (graus IV e V), quando presentes, eram decorrentes de acidente por veículo auto-motor e que, esse mesmo mecanismo de trauma, foi também o responsável pela maioria das lesões mais leves (graus I, II e III). Em virtude da ausência de estudos a esse respeito, não há dados na literatura para se comparar com os encontrados neste trabalho.

O índice de suspeita para lesão abdominal deve ser baseado no mecanismo de trauma e nos achados de exame físico, como a presença de dor abdominal ${ }^{11}$. Portanto, é de grande importância saber se o paciente traumatizado manifesta ou não esse sintoma. Neste trabalho, a maioria dos pacientes $(74,24 \%)$ com lesão hepática apresentou dor abdominal, não havendo diferença estatística entre os distintos graus de lesão (Tabela 5). Infelizmente, não há dados na literatura que relacionem dor abdominal e grau de lesão hepática.

Estima-se que haja fratura de costelas em aproximadamente $10 \%$ dos pacientes vítimas de trauma fechado ${ }^{12}$, sendo que a fratura de arcos costais baixos $\left(9^{\circ}\right.$ a $\left.12^{\circ}\right)$ está comumente associada à lesão de órgãos como fígado, baço ou rim $^{12,13}$. Em estudo de Shweiki et al. ${ }^{14}$, a incidência de lesão

Tabela 6 - Média da freqüência cardíaca e da pressão arterial sistólica de entrada de acordo com o grau de lesão hepática.

\begin{tabular}{l|lcc|ccc}
\hline Grau de lesão hepática & \multicolumn{3}{|c|}{ FC } & \multicolumn{3}{c}{ PA sistólica } \\
\hline & $\mathrm{n}$ & média & $\mathrm{dp}$ & $\mathrm{n}$ & média & $\mathrm{dp}$ \\
I ou II & 30 & 100,37 & 17,65 & 29 & 114,45 & 25,46 \\
III, IV ou V & 26 & 105,69 & 22,07 & 25 & 107,88 & 25,60 \\
\hline
\end{tabular}

$F C=$ freqüência cardíaca $; P A=$ pressão arterial $; d p=$ desvio padrão

$p=0,3204(F C)$

$p=0,3504$ (PA sistólica) 
hepática em pacientes com fratura de costelas foi de $10,7 \%$. Neste trabalho, observou-se que $29,41 \%$ dos pacientes com fratura de costelas à direita apresentaram lesão hepática associada, sendo que todas as lesões eram grau I ou II.

Sabe-se que o indicador mais confiável de sangramento intra-abdominal é a presença de choque de origem não explicada. Esta é a razão pela qual é imperativo reconhecer os seus sinais mais precoces, como a taquicardia ${ }^{11}$. Neste estudo, observou-se que os pacientes vítimas de trauma hepático grave apresentaram, em média, mais taquicardia que aqueles com lesão mais leve (Tabela 6). Entretanto, essa diferença não foi estatisticamente significativa.

Apesar da pressão arterial sistólica ser um indicador tardio no reconhecimento do estado de choque ${ }^{11}$, é um parâmetro relevante na avaliação da perda estimada de líquido e sangue e da reposição volêmica ${ }^{15}$. No presente trabalho, verificou-se que a pressão arterial sistólica no momento da admissão do paciente ao hospital não apresentou diferença estatística de acordo com o grau da lesão hepática (Tabela 6).
Considerando os dados obtidos, pode-se afirmar que:

- Os acidentes por veículo automotor foram a causa mais comum de trauma hepático e o mecanismo de trauma que provocou as lesões mais graves (graus III, IV e V);

- As lesões hepáticas mais leves (grau I, II e III) foram as mais prevalentes;

- As lesões hepáticas mais graves causaram dor abdominal com a mesma frequiência que as mais leves;

- A pressão arterial sistólica e a frequiência cardíaca média, no momento da admissão do paciente ao hospital, não apresentaram relação com o grau da lesão hepática;

- A fratura de arcos costais à direita influenciou apenas na presença da lesão hepática, não possuindo relação com o grau das lesões.

Em virtude da pouca literatura a respeito das características do trauma hepático, sugere-se que mais estudos sejam realizados para que, assim, seja possível estabelecer protocolos e condutas mais apropriados à realidade do Sistema Único de Saúde.

\begin{abstract}
Background: To determine during hospital admissions, the trauma mechanism, anatomical and physiological injuries, abdominal pain and rib fractures of hepatic trauma victims. Methods: Records from December/1999 to January/2003 were reviewed and 524 were selected by the Software TNVT Plus (version 2.0.0.213-year 1996), from patients admitted to the Emergency Service, victims of blunt thoracic-abdominal trauma and submitted partial hepatectomy, hepatorrhaphy, laparotomy, reduction or conservative treatment of rib fractures. Those records which didn't present blunt trauma, were excluded, as well as the ones who deceased in the emergency room and those under 16 years old. Therefore, 200 records were analysed. Results: Regarding all liver traumas, 37.63\% were caused by motor vehicle accidents, responsible for 54.29\% of the most serious injuries (degrees III, IV and V). These injuries caused abdominal pain in $75.86 \%$ of the cases, and minor injuries (degrees I and II) caused abdominal pain, in 69.70\%. The heart rate and the mean systolic arterial pressure with degree I or II injuries were, respectively, 100.37 beats per minute and $114.45 \mathrm{mmHg}$; and in those with degree III, IV, or Vinjuries were 105.69 beats per minute and $107.88 \mathrm{mmHg}$, respectively. Right rib fracture was associated to degrees I or II injuries in $29.41 \%$ of the cases. Conclusion: Motor vehicle accidents are the most common cause of hepatic trauma and is the main causes of the most serious injuries. These injuries caused abdominal pain with the same frequency that minor injuries. The heart rate and mean systolic arterial pressure showed no relation to degree of hepatic injury.
\end{abstract}

Key words: Abdominal injuries; Liver/ injuries; Wounds and injuries.

\section{REFERÊNCIAS}

1. Gür S, Orsel A, Atahan K, et al. Surgical treatment of liver trauma (analysis of 244 patients). Hepatogastroenterology. 2003;50(54):2109-11.

2. Carrillo EH, Platz A, Miller FB, et al. Non-operative managment of blunt hepatic trauma. Br J Surg.1998;85(9):461-8.

3. Zantut LFC, Poggetti RS, Birolini D. Tratamento conservador de trauma hepático grave: relato de caso e revisão de métodos terapêuticos. Rev Hosp Clín Fac Med São Paulo. 1993;48(5):23541.

4. Romano L, Giovine S, Guidi G, et al. Hepatic trauma: CT findings and considerations based on our experience in emergency diagnostic imaging. Eur J Radiol. 2004; 50(1):59-66.

5. Letoublon $\mathrm{C}$, Arvieux C. Nonoperative management of blunt hepatic trauma. Minerva Anestesiol. 2002;68(4):132-7.

6. Velmahos GC, Toutouzas K, Radin R, et al. High success with nonoperative management of blunt hepatic trauma: the liver is a sturdy organ. Arch Surg.2003; 138(5):475-81.
7. Veroux M, Cillo U, Brolese A, et al. Blunt liver injury: from nonoperative management to liver transplantation. Injury. 2003;34(3):181-6.

8. Hsieh $\mathrm{CH}$, Chen RJ, Fang JF, et al. Liver abscess after nonoperative management of blunt liver injury. Langenbecks Arch Surg. 2003;387(9-10):343-7.

9. Croce MA, Fabian TC, Menke PG, et al. Nonoperative management of blunt hepatic trauma is the treatment of choice for hemodynamically stable patients. Results of a prospective trial. Ann Surg.1995;221(6):744-55.

10. Pachter HL, Knudson MM, Esrig B, et al. Status of nonoperative management of blunt hepatic trauma in 1995: a multicenter experience with 404 patients. J Trauma. 1996;40(1):31-8.

11. Atendimento pré-hospitalar ao traumatizado: básico e avançado. Comitê do PHTLS da National Association of Emergency Medical Technicians (NAEMT) em cooperação com o Comitê de Trauma do Colégio Americano de Cirurgiões; [tradutores: Renato Sérgio Poggetti et al] $-5^{\mathrm{a}}$ ed. Rio de Janeiro: Elsevier; 2004. 
12. Moore EE, Mattox KL, Feliciano DV. Trauma manual. $4^{\text {th }}$ ed. New York: McGraw-Hill; 2003.

13. Nadalo LA, Jones K. Rib fractures. eMedicine [serial on the Internet]. 2005 june. Available from: http://www.emedicine.com/ emerg/topic204.htm.

14. Shweiki E, Klena J, Wood GC, et al. Assessing the true risk of abdominal solid organ injury in hospitalized rib fracture patients. J Trauma. 2001;50(4):684-8.

15. Suporte avançado de vida no trauma. Comitê de Trauma do Colégio Americano de Cirurgiões. $6^{a}$ ed. 1997.
Endereço para correspondência:

Carlos Andrés Rodriguez Pantanali

Av. Dr. Carlos de Carvalho, 1461 - apto. 45B.

Cep: $80-730200$

Bairro: Batel

Curitiba-PR

e-mail: capush_mail@yahoo.com.br 